\title{
Phototropism and Light-growth Responses of the Tall Conidiophores of Aspergillus giganteus
}

\author{
By A. P. J. TRINCI AND G. H. BANBURY \\ Department of Microbiology, Queen Elizabeth College (University of \\ London), Campden Hill Road, London, W. 8 and Department of Botany, \\ University of Durham
}

(Accepted for publication 7 August 1968)

\section{SUMMARY}

Aspergillus giganteus conidiophores are positively phototropic to unilateral stimulation with white light. The response is restricted to the apical $240 \mu$ of the conidiophore. The curvatures start about $22 \mathrm{~min}$. after the onset of stimulation and proceed at a rate of about $2.5^{\circ}$ min.-1. During the response the rate of extension of the proximal wall (with respect to the direction of the incident illumination) decreases by about $5 \%$ as compared to its previous rate of growth, whilst that of the distal wall increases by about $5 \%$; thus there is no net change in the rate of wall growth during phototropism. The sign of the phototropic response is reversed when conidiophores are unilaterally stimulated with ultraviolet radiation $(280 \mathrm{~m} \mu)$ or with white light whilst submerged in liquid paraffin. Conidiophores cultured on media containing l-lyxoflavin or diphenylamine show normal phototropic responses.

A tenfold increase or decrease in light intensity is followed after approximately $12 \mathrm{~min}$. by a slight change (about I I \%) in the rate of conidiophore growth. However, changes in light intensity between I 39 and 268 lux do not affect the growth rate. There is an acceleration in growth rate when conidiophores previously held in red light for some hours are re-illuminated with white light.

\section{INTRODUCTION}

Although most fungal hyphae are insensitive to unilateral stimulation with white light there are some, particularly those which bear reproductive structures, which are phototropic. These hyphae are usually positively phototropic but negative responses have also been reported (Welty \& Christensen, 1965). The selective advantage of reproductive structures being positively phototropic is presumably because spore dispersal is directed towards what is usually open space.

A second light-induced response which has been studied in fungi is the so-called light-growth response. A light-growth response may be defined as a change in growth rate in response to a change in light intensity. When growth is accelerated on increasing the light intensity, the light-growth response is said to be positive, whilst when there is a decrease in growth rate the response is said to be negative.

The hyphae most frequently chosen for studying phototropism and light-growth responses in fungi are the sporangiophores of Phycomyces blakesleeanus. The advantage of using this species is that its sporangiophores are large, robust and relatively xerophytic. As a result of intensive investigation the growth kinetics and pattern of wall extension of $P$. blakesleeanus sporangiophores during phototropic and light- 
growth responses have been precisely defined (Shropshire, 1963). However, this study has not led to full understanding of the nature of the perceptive and response mechanisms involved. Much more needs to be known about normal features of hyphal growth before the nature of the changes induced by external stimuli can be fully understood.

There are some advantages in using Phycomyces blakesleeanus sporangiophores to study phototropic and light-growth responses but there is also a need to investigate these phenomena in other species. To a certain extent this has already been done (Banbury, 1959; Carlile, 1965). The advantage of using Aspergillus giganteus is that phototropism can be studied in the absence of geosensitivity (Trinci \& Banbury, 1967) and that conidiophore elongation, unlike the growth of $P$. blakesleeanus sporangiophores, is a light dependent process; $P$. blakesleeanus sporangiophores grow at the same rate in continuous darkness or light, but frequent light stimuli are essential if the elongation of $A$. giganteus conidiophores is to be maintained (Trinci \& Banbury, in press). In previous studies on Aspergillus giganteus we have described the kinetics of conidiophore growth, the influence of light on conidiophore production and carotenogenesis and the ultrastructural details of sporulation (Trinci \& Banbury, 1967; Trinci, Peat \& Banbury, I968; Trinci \& Banbury, 1969).

\section{METHODS}

Strain W-267 of Aspergillus giganteus from Professor A. Weston's collection at Harvard University was used. The fungus was cultured on Oxoid malt extract agar except when the action of I-lyxoflavin or diphenylamine were being tested, in which case the the following medium was used (g./1.): D-glucose, 50.0; $\mathrm{KH}_{2} \mathrm{PO}_{4}, \mathrm{I} \cdot 0$; $\mathrm{NH}_{4} \mathrm{NO}_{3}, \mathrm{I} \cdot 0 ; \mathrm{MgSO}_{4} \cdot 7 \mathrm{H}_{2} \mathrm{O}, \mathrm{I} \cdot 0 ; \mathrm{ZnSO}_{4} \cdot 7 \mathrm{H}_{2} \mathrm{O}, 0.002 ; \mathrm{FeSO}_{4} \cdot 7 \mathrm{H}_{2} \mathrm{O}$, 0.002. When diphenylamine was added to this medium the glucose concentration was decreased from 5 to $\mathrm{I} \%$. The media were sterilized by autoclaving for $15 \mathrm{~min}$. at $12 \mathrm{I}^{\circ}$.

Microscopic studies of conidiophore growth were made on cultures growing in observation cells (P1. I, fig. I) under the conditions described by Trinci \& Banbury, I967, or in the apparatus shown in Fig. I. The red observation light was on for the duration of each experiment; tall conidiophores were not produced when it was the sole source of illumination and no phototropic response was induced when it was used to illuminate conidiophores unilaterally.

Conidiophore growth was followed by time-lapse photography, with measurements made on tracings taken of the projected film (final magnification $\times 335$ ), or by direct measurement with a micrometer eyepiece (one division of the micrometer equalled $0.83 \mu$ ). The concave and convex flanks of curved conidiophores were measured by 'walking' dividers along their lengths and adding up the secant distances. The length of the apical phototropically sensitive segment of the conidiophore was estimated by superimposing the outlines of responding conidiophores traced from successive photographs.

The intensity of the white light illuminating cultures was measured in lux by using an SEI exposure photometer or a Weston Master IV exposure meter (both supplied by Ilford Ltd., London). One lux of white cycle $=\mathrm{I} \cdot 6 \mathrm{erg} . / \mathrm{cm} .^{2}$ (Westphal, 1947).

Cultures were initially incubated for 2 to 3 days at $22^{\circ} \pm I^{\circ}$ in an illuminated incubator and then transferred to the horizontal microscope for observation. All experiments 
were made in a dark room at $23.0 \pm 0.5^{\circ}$ on conidiophores which were 4 to $8 \mathrm{~mm}$. tall, i.e. they were in the linear phase of growth (Trinci \& Banbury, 1967).

The effect on growth rate of altering the light intensity between 20 and 200 lux was studied by using conidiophores which had been grown for 20 to $26 \mathrm{hr}$ at one or other light intensity. In these experiments the effect on growth rate of either one step up or step down in light intensity was followed. When the light intensity illuminating the
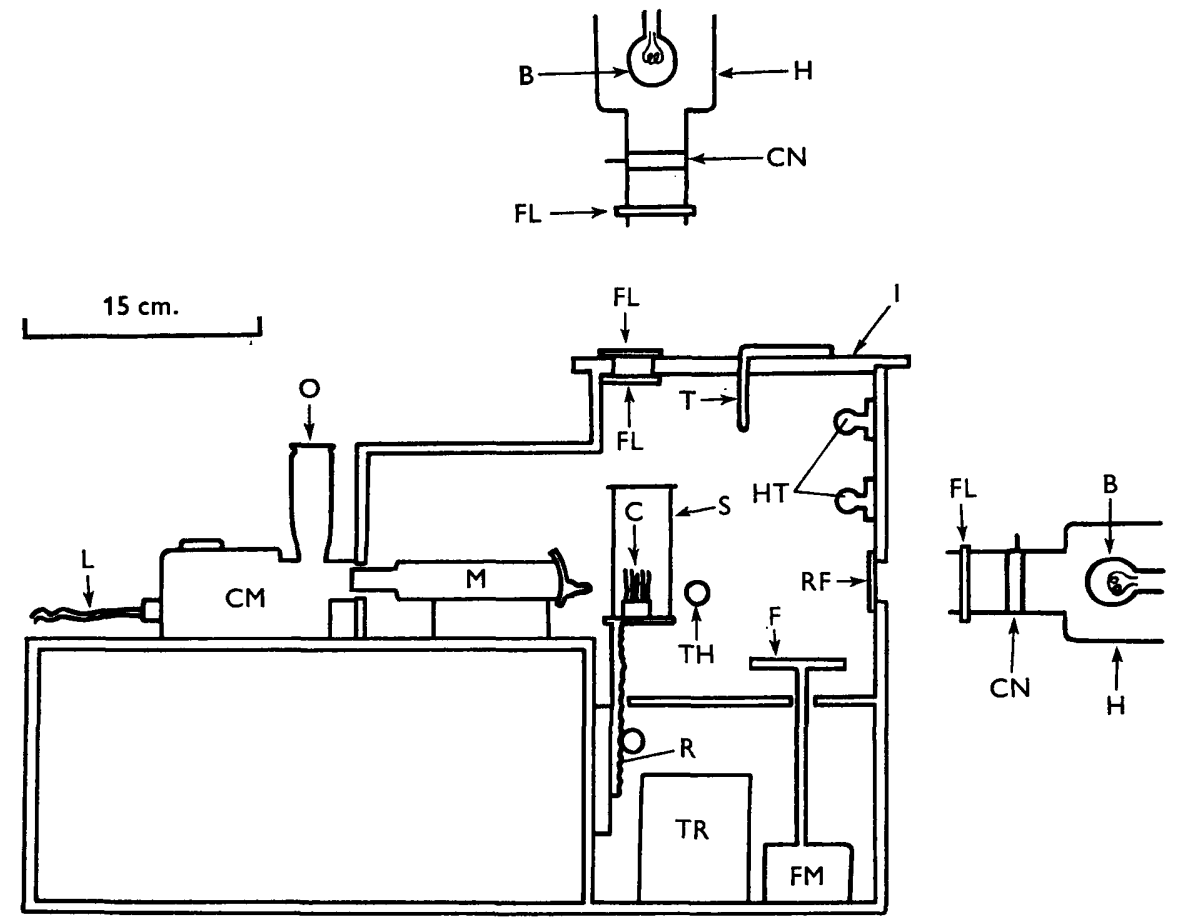

Fig. I. Apparatus used to follow conidiophore growth and phototropism.

B $\quad 6 \mathrm{~V}, 48 \mathrm{~W}$ tungsten filament bulb

C Conidiophores

CM Camera

CN Condenser

F Fan

FL Infra red absorbing glass filter

FM Fan motor

H High intensity lamp connected to rheostat

HT Incubator heat source (blacked out cycle bulbs)

I Wooden incubator
L Lead to intervalometer

M Microscope barrel, objective and eyepiece

O Observation eyepiece

R Rack for vertical movement of specimen

RF Red filter emitting no light below $620 \mathrm{~m} \mu$

S Specimen chamber

T Thermometer

TH Thermostat barrel

TR Transformer supplying cycle bulbs

cultures was varied between 139 and 268 lux, each conidiophore was subjected to four successive changes in light intensity at hourly intervals.

When the phototropic response was studied, the conidiophores were first illuminated with white light from above and then this light was switched off and the cultures were illuminated from one side. Statistical analysis of the experimental results was based on formulae from Moroney (1956). 


\section{RESULTS}

Influence of changes in light intensity on the rate of conidiophore elongation

The effect on the rate of conidiophore growth of a step up or a step down in light intensity between 20 and 200 lux was determined. Statistical analysis of the results (Table $\mathrm{I}$ ) indicated that about $\mathrm{I} 2 \mathrm{~min}$. after the increase or decrease in light intensity there was a significant change in the rate of conidiophore growth. In each case the new rate of growth was persistent during the I to $2 \mathrm{hr}$. period of observation.

Table I. Statistical comparison between the initial rate of conidiophore growth and the subsequent rates after altering the light intensity

Measurements of conidiophore growth were taken at 2 min. intervals. Statistical comparison $_{\mathrm{S}}$ were based on data obtained during successive $4 \mathrm{~min}$. intervals.

\begin{tabular}{|c|c|c|}
\hline \multirow{2}{*}{$\begin{array}{l}\text { Time since } \\
\text { the light } \\
\text { intensity } \\
\text { was changed } \\
\text { (in min.) }\end{array}$} & \multicolumn{2}{|c|}{$\begin{array}{l}\text { Light intensity decreased from } \\
200 \text { to } 20 \text { lux. Mean growth rate } \\
\text { at } 200 \text { lux }=8.7 \mu / \mathrm{min} \text {. }\end{array}$} \\
\hline & $\begin{array}{l}\text { Mean rate } \\
\text { of growth } \\
(\mu / \mathrm{min} .)\end{array}$ & $\begin{array}{l}\text { Probability } \dagger \\
\text { (Student's t)* }\end{array}$ \\
\hline 4 & $8 \cdot 7$ & - \\
\hline 8 & $8 \cdot 5$ & $<0.4$ \\
\hline 12 & $8 \cdot 3$ & $<0.2$ \\
\hline 16 & $8 \cdot 0$ & $<0.05$ \\
\hline 20 & $7 \cdot 6$ & $<0.01$ \\
\hline 24 & $7 \cdot 6$ & $<0.01$ \\
\hline 28 & $7 \cdot 8$ & $<0.02$ \\
\hline 32 & 8.0 & $<0.05$ \\
\hline 36 & $8 \cdot I$ & $<0.10$ \\
\hline 40 & $7 \cdot 8$ & $<0.02$ \\
\hline 44 & $7 \cdot 8$ & $<0.02$ \\
\hline 48 & $7 \cdot 8$ & $<0.02$ \\
\hline 52 & $8 \cdot 0$ & $<0.10$ \\
\hline 56 & $8 \cdot 0$ & $<0.10$ \\
\hline 60 & $7 \cdot 9$ & $<0.05$ \\
\hline
\end{tabular}

Light intensity increased from 20 to 200 lux. Mean growth rate at $20 \operatorname{lux}=6 \cdot 7 \mu / \mathrm{min}$.

$\overbrace{\begin{array}{c}\text { Mean rate } \\ \begin{array}{c}\text { of growth } \\ (\mu / \mathrm{min})\end{array}\end{array} \quad \begin{array}{c}\text { Probability }{ }^{\dagger} \\ \text { (Student's } t)^{*}\end{array}}$

$\begin{array}{ll}6.3 & <0.2 \\ 6.7 & - \\ 6.8 & <0.7 \\ 7.3 & <0.1 \\ 7.7 & <0.01 \\ 7.4 & <0.01 \\ 7.5 & <0.01 \\ 7.1 & <0.05 \\ 7.0 & <0.2 \\ 7.1 & <0.05 \\ 7.5 & <0.01 \\ 7.7 & <0.01 \\ 8.0 & <0.01 \\ 8.0 & <0.01 \\ 7.7 & <0.01\end{array}$

$\dagger$ Probability of the difference between the mean growth rates before and after change in light intensity resulting from chance variation.

* Student's $t 0.05=$ difference probably significant, $0.0 \mathrm{I}=$ significant difference, $0.00 \mathrm{I}=$ highly significant difference.

The mean rate of growth of the eight conidiophores cultured at 200 lux was $8.7 \mu /$ min. (standard deviation $\pm \mathrm{I} \cdot 32 \mu$ ) whilst their mean rate of growth from a period $\mathrm{I} 2 \mathrm{~min}$. after the decrease in light intensity was $7.9 \mu / \mathrm{min}$.; thus there was on darkening an II \% decrease in growth rate. The mean rate of growth of the five conidiophores cultured at 20 lux was $6.7 \mu / \mathrm{min}$. (standard deviation $\pm 0.56 \mu$ ), whilst the mean rate of growth from a period I $2 \mathrm{~min}$. after increasing the light intensity was $7 \cdot 5 \mu / \mathrm{min}$.; thus in this case there was about a $12 \%$ increase over the previous growth rate.

We previously reported that the degree of apical oscillation of the conidiophore about its vertical axis of growth was greater at 20 lux than at 200 (Trinci \& Banbury, I967). This variation is oscillation with light intensity (Pl. I, fig. 2, 3) may have resulted in an underestimation of the rate of growth at the lower intensity used (in 
these experiments measurements of growth were made with the micrometer eyepiece). In view of this possibility it was decided to determine the effect on growth rate of changing the light intensity between $\mathrm{I} 39$ and $268 \mathrm{lux}$. In this case the degree of conidiophore oscillation was slight even at the lower intensity and it was thus less likely that a variation in the degree of oscillation would cause a significant error in the measurement of growth rate. The effect on conidiophore growth of a step up or a step down in light intensity between 139 and 268 lux is shown in Fig. 2.

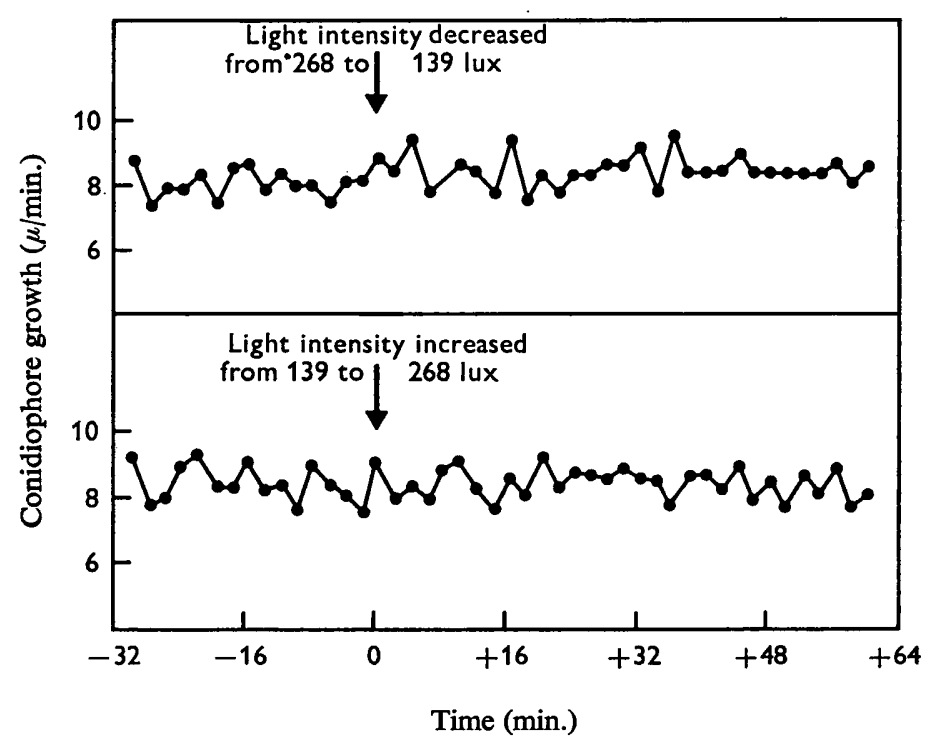

Fig. 2. Influence of an increase or decrease in light intensity between 139 and 268 lux on the rate of conidiophore growth. Each graph represents the mean of five changes in light intensity. Light intensity changes at arrow.

The results were analysed statistically in the same way as in the previous experiment. There was no significant change in the rate of conidiophore growth when the light intensity was either increased or decreased. For the step down in light intensity the calculated probabilities (probability of the difference between the mean growth rates before and at $4 \mathrm{~min}$. intervals after the change in light intensity resulting from chance variation) ranged from 0.2 to 0.8 whilst for the step up in light intensity they ranged from 0.3 to 0.9 . Thus in these experiments the conidiophores did not show a positive growth response on increasing the light intensity or a negative growth response on darkening. The light-growth response of the sporangiophores of Phycomyces blakesleeanus (taken from Delbrück \& Reichardt, 1956) and Thamnidium elegans (taken from Lythgoe, 196I) and the conidiophores of Aspergillus giganteus are presented in Fig. 3 so that they may be compared. To our knowledge these are the only three species in which the light-growth response of positively phototropic hyphae has been investigated. 


\section{Light-growth response and phototropism of conidiophores grown for a period in red light}

In a previous paper (Trinci \& Banbury, 1967) we described the effect of white light on conidiophores whose rate of growth had decreased following a $24 \mathrm{hr}$ period in red light. After a lag of about $30 \mathrm{~min}$. the rate of conidiophore growth increased slowly from about $4 \mu / \mathrm{min}$. to about $7.5 \mu / \mathrm{min}$. In the present experiments conidiophores

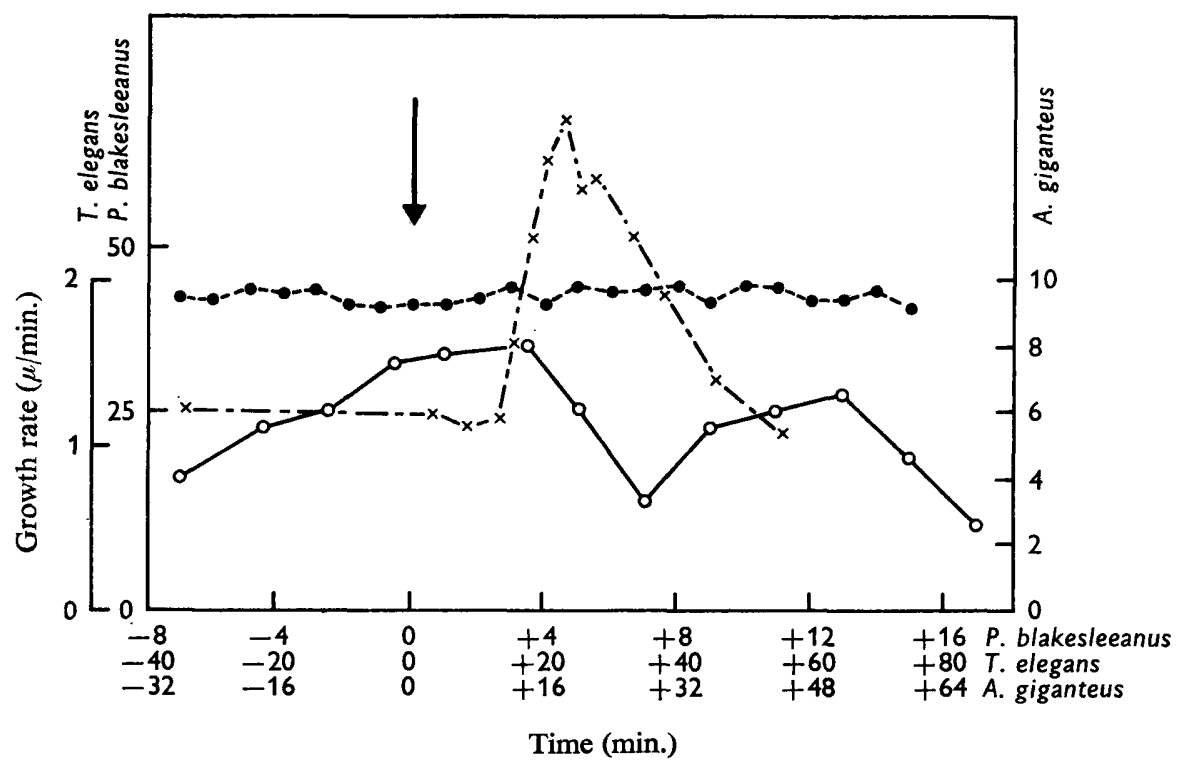

Fig. 3. Comparison between the light-growth responses of Phycomyces blakesleeanus (from Delbrück \& Reichardt, 1956), Thamnidium elegans (from Lythgoe, 196I) and Aspergillus giganteus. $P$. blakesleeanus, $\times-\times$, transient increase in light intensity at arrow. T. elegans, $\mathrm{O}-\mathrm{O}$, change from darkness to light at arrow. A. giganteus, - - $--\infty$, step up in light intensity from 139 to 268 lux at arrow.

which had been grown for $15 \mathrm{hr}$ in red light were unilaterally illuminated with white light (200 lux); $35 \mathrm{~min}$. later the conidiophore started to respond with a positive phototropic curvature. The curvature, which proceeded at a rate of about $3.5 \% \mathrm{~min}$. was completed some $20 \mathrm{~min}$. later. The change in rate of growth of the conidiophore wall during phototropism is presented in Table 2 and Fig. 4. During the phototropic response there was a $41 \%$ increase in the mean rate of wall extension as compared to the original growth rate and there was a difference of about $35 \%$ between the growth rates of the distal and proximal walls. After completion of the phototropic response there was a decrease in the extension rate.

This type of response occurred in the three conidiophores which were unilaterally stimulated in this manner, although there was not always as great an increase in growth rate during phototropism. In the case of the conidiophore drawn in Fig. 4, the apical $200-225 \mu$ of the conidiophore responded to the unilateral stimulus; this is almost exactly the length calculated previously for the extension zone (Trinci \& Banbury, 1967) and would seem to indicate that the decrease in growth rate which occurred in red light or darkness was not accompanied by a shortening of the extension zone. 


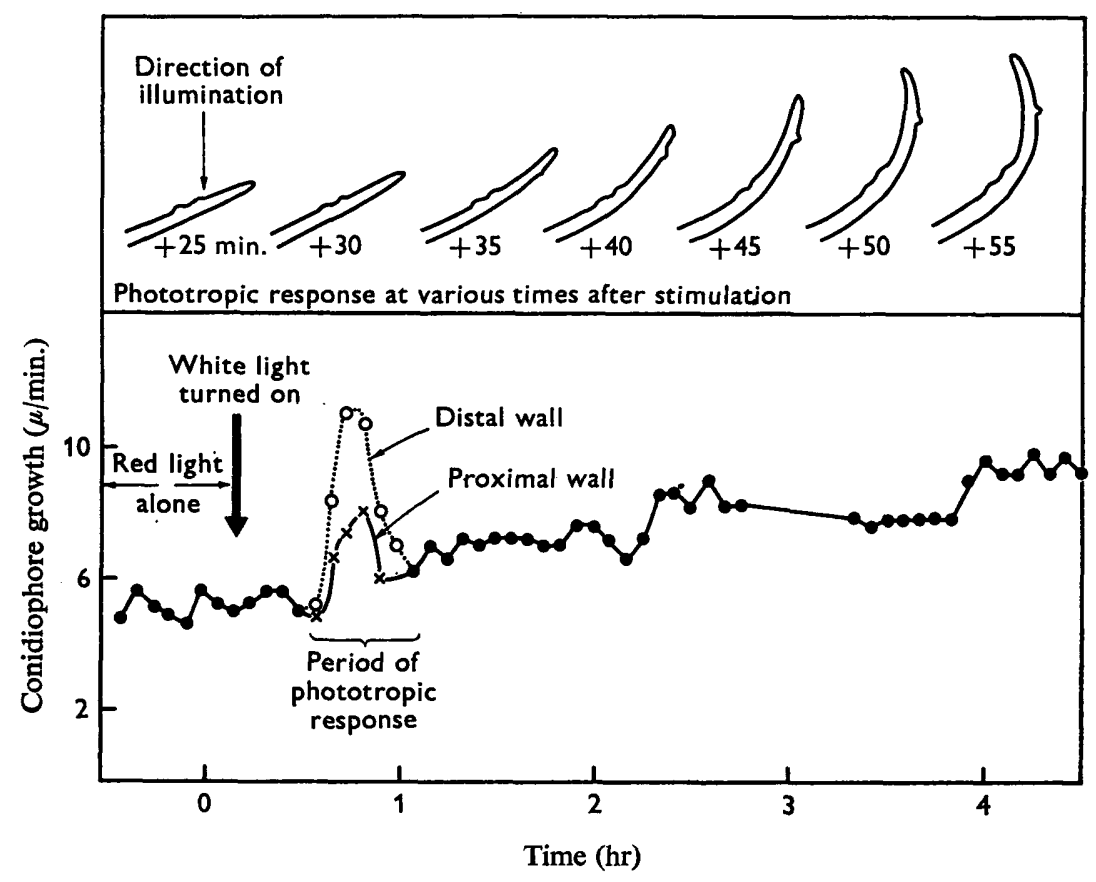

Fig. 4. Influence of white light on the rate of growth and phototropism of a unilaterally illuminated conidiophore previously held in red light for $15 \mathrm{hr}$. Illuminated with white light at arrow.

Table 2. Changes in the rate of wall growth during the phototropic response of a conidiophore previously held in red light for $15 \mathrm{hr}$

$\begin{array}{cc}\text { Mean growth } & \begin{array}{c}\text { Growth as a } \\ \% \text { of rate } \\ \text { prior to the } \\ \text { response } \\ (5.4 \mu / \text { min. })\end{array} \\ \text { rate }(\mu / \text { min. }) & 100 \\ 5.4 & \\ 8.8 & 163 \\ 6.5 & 120 \\ 7.6 & 142 \\ 6.9 & 128\end{array}$

The above experiments confirmed the fact that there is an acceleration in growth when conidiophores held in red light for some hours are re-illuminated with white light. However, it is clear that this response is essentially different from the positive light-growth response of Phycomyces blakesleeanus sporangiophores. In P. blakesleeanus an increase in light intensity (Fig. 3) or a change from darkness to light has only a transitory stimulatory effect on the rate of sporangiophore elongation but in Aspergillus giganteus continued growth of the conidiophores is dependent upon frequent white light stimuli (Trinci \& Banbury, 1969). 


\section{Phototropic response to white light in air}

Conidiophores which had previously been illuminated from above were photographed at $2 \mathrm{~min}$. intervals before and after unilateral stimulation with white light. The response of five conidiophores treated in this manner was analysed. The mean lag after stimulation before the onset of the response was $22 \mathrm{~min}$. The mean length of the apical segment of the conidiophore which responded to the unilateral stimulus was 24I $\mu$ (standard deviation $\pm 4 \mathrm{I} \mu$ ) and the phototropic curvature proceeded at a mean rate of $2.5^{\circ} / \mathrm{min}$. (standard deviation $\pm 0.5^{\circ}$ ). The region of the conidiophore which responds phototropically is restricted to the extension zone. We previously reported that experiments in which starch grains were dusted on to the condiophore indicated that the extension zone varied between 170 and $240 \mu$ (Trinci \& Banbury, 1967). There is thus quite good agreement between the two methods used to determine the length of the extension zone.

The mean rate of growth of the conidiophores before unilateral stimulation and the rates of growth of the proximal and distal walls (with respect to the direction of incident illumination) during the response are presented in Table 3. The rate of extension of the distal wall accelerated by $5.3 \%$ during the phototropic curvature, whilst that of the proximal wall decreased by $4.5 \%$. The mean between the rates of extension of the distal and proximal walls was almost exactly the same as the growth rate of the conidiophore before stimulation. Thus during the phototropic response there was a redistribution of growth within the extension zone but apparently no net increase or decrease in the rate of wall extension.

Table 3. Change in the rate of wall growth during phototropism

\begin{tabular}{|c|c|c|}
\hline Mear & nidiophores & \\
\hline & $\begin{array}{l}\text { Mean growth rate } \\
(\mu / \mathrm{min} .)\end{array}$ & $\begin{array}{l}\text { Growth as } \% \text { of } \\
\text { original rate } \\
(10.04 \mu / \mathrm{min} .)\end{array}$ \\
\hline $\begin{array}{l}\text { I. Growth prior to unilateral } \\
\text { stimulation }\end{array}$ & $10.04( \pm 0.82)^{*}$ & 100 \\
\hline $\begin{array}{l}\text { 2. Growth during the photo- } \\
\text { tropic response }\end{array}$ & & \\
\hline (a) Distal wall & $10.57( \pm 0.59)^{*}$ & $105 \cdot 3$ \\
\hline (b) Proximal wall & $9.59( \pm 0.82)^{*}$ & 95.5 \\
\hline $\begin{array}{l}\text { (c) Mean of proximal and } \\
\text { distal walls (axis) }\end{array}$ & 10.08 & $100 \cdot 4$ \\
\hline
\end{tabular}

The response of conidiophores to unilateral illumination is to some degree dependent upon their previous history. The rate of phototropic curvature was faster when the conidiophores had been held in red light before stimulation than when they had previously been illuminated with white light.

\section{Phototropic response to ultraviolet light and under liquid paraffin}

A phototropic response is induced by the establishment of a light gradient across the hypha; when the radiation intensity is greater on the distal side of the hypha (with respect to the direction of incident illumination) the tropic response is positive; when it is greater on the proximal side, the tropic response is negative. The side which re- 
ceives the greater illumination is determined by which of the optical properties of the hypha predominates, the 'lens effect' (the hypha behaving as a cylindrical lens focusing light on the rear wall) or attenuation of light within the hypha. These two properties of the sporangiophore work in opposition; the 'lens effect' tends to promote positive tropic curvatures whilst severe attenuation of the light across the cytoplasm results in negative curvatures.

Two experiments were undertaken to determine whether the optical system operative in Aspergillus giganteus conidiophores is the same as that found in Phycomyces blakesleeanus sporangiophores (Shropshire, 1963). To determine if the 'lens effect' is operative the conidiophores were submerged in liquid paraffin and unilaterally illuminated with white light. All the conidiophores which continued to grow after this treatment responded to the stimulation with sharp negative phototropic curvatures (Pl. I, fig. 4). The formation of droplets along the wall continued during the period in which the conidiophores grew in liquid paraffin, demonstrating that they arose by exudation of material from within the conidiophore rather than by condensation.

The effect of light attenuation across the conidiophore was demonstrated by unilaterally stimulating conidiophores with ultraviolet radiation of wavelength $280 \mathrm{~m} \mu$; a spectrophotometer was used as the source of monochromatic ultraviolet radiation. The conidiophores responded to the unilateral stimulus by sharp negative phototropic curvatures (Pl. I, fig. 5). Curry \& Gruen (1957) showed that the tropic response of Phycomyces blakesleeanus sporangiophores was negative for any wavelength shorter than $302 \mathrm{~m} \mu$. In this case the negative curvature is due to strong absorption of the short wavelength radiation on the side of the sporangiophore nearest to the light source, probably by gallic acid (Dennison, 1959).

\section{Action of l-lyxoflavin and diphenylamine}

Riboflavin and carotene have both been considered as the photoreceptive pigment in fungal phototropism (Carlile, 1965). Page (1956) found that 1-lyxoflavin, a riboflavin analogue, inhibited the photoinduction of trophocyst formation in Pilobolus and interpreted the results as indicating that riboflavin functions as the photoreceptor in this process. The effect of 1-lyxoflavin on the phototropic response of Aspergillus giganteus conidiophores was therefore examined and also that of diphenylamine which strongly inhibits the synthesis of carotene in fungal mycelium when added to the culture medium. A. giganteus was grown on media to which one or other of these two inhibitors was added and the effect on the reaction time before initiation of a phototropic response studied. The results are presented in Table 4 . The fact that conidiophores took longer to respond on the medium containing diphenylamine was probably a reflection of the slower growth of the fungus rather than to any selective inhibition of the photoreceptive mechanism ( $40 \mu \mathrm{g}$. diphenylamine/ml. of medium decreased the radial growth rate of colonies by about $40 \%$ ). Gressel \& Hartmann (1968) reported that incorporation of 1-lyxoflavin and diphenylamine in the culture medium inhibited the growth of Trichoderma viride to a greater extent than the photoinduction of sporulation. 
Table 4. Effect on the duration of the reaction time of the phototropic response of the addition of l-lyxoflavin and diphenylamine to the medium

\author{
Period between \\ onset of unilateral \\ stimulation and \\ onset of the photo- \\ tropic response \\ (min.)
}

Treatment

I. Control*

2. L-Lyxoflavin (100 $\mu \mathrm{g} . / \mathrm{ml}$.) added to medium

3. Diphenylamine ( $40 \mu \mathrm{g} . / \mathrm{ml}$.) added to medium
33

32

42

* The longer reaction time of the conidiophores in these experiments as compared with those described previously is probably due to the fact that a different medium was employed.

\section{DISCUSSION}

There appear to be two distinct responses in Aspergillus giganteus to light. First, light is essential for the maintenance of conidiophore elongation and causes an acceleration in the growth of conidiophores previously held in red light or darkness for some hours, and secondly, asymmetrical illumination causes a redistribution of the growth potential and results in phototropism. This conclusion seems to be supported by experiments in which it was shown that by varying the frequency of illumination the influence of light in inducing conidiophore growth could be divorced from its orientating effect; illumination of cultures for $12 \mathrm{~min}$./12 hr or 10 $\mathrm{sec}$./ $10 \mathrm{~min}$. (same total light dose) resulted in the induction of the same amount of conidiophore growth (as measured on a dry-weight basis) but only under the latter light régime were the conidiophores orientated with respect to the direction of the incident illumination (Trinci \& Banbury, 1969).

The fact that negative phototropic curvatures result both from unilateral stimulation of conidiophores submerged in liquid paraffin in white light, and with ultraviolet radiation in air, demonstrates that the optical properties of Aspergillus giganteus conidiophores are essentially the same as those of Phycomyces blakesleeanus sporangiophores. Unilateral illumination with white light in air results in the establishment of a light gradient across the conidiophore which in turn causes a redistribution of the growth potential within the hypha (as distinct from any net stimulation of growth) and hence a phototropic curvature. Under our experimental conditions the rate of growth of the distal wall is increased by about $5 \%$ during phototropism whilst that of the proximal wall is depressed by about the same amount.

The light-growth responses of the three positively phototropic species which have been examined to date do not show a common pattern (Fig. 3). As far as Aspergillus giganteus is concerned, it seems that at least for small changes in light intensity there is no significant change in growth rate. The slight change in growth rate recorded for a tenfold increase in light intensity may not represent a true acceleration in growth for the reasons already stated. Thus it does not seem possible to explain phototropism in A. giganteus on the basis of unequal light-growth responses on the 'near' and 'far' sides of the curving conidiophore. Even in Phycomyces blakesleeanus it has proved 
possible to induce a phototropic curvature without any light-growth response (Castle, I96I $a$ ). In the case of Thamnidium elegans, Lythgoe (196I) found that a positive phototropic response is coupled with a negative light-growth response (Fig. 3). He suggested that this apparent contradiction could be explained by the fact that the onset of the phototropic response coincides in time with the recovery phase of the negative light-growth response. However, Lythgoe did not conduct the critical experiment which might have substantiated this hypothesis, viz. comparing the recovery rates in light and in darkness after an initial period in light during which the growth rate was reduced.

At this stage it is only possible to speculate on the nature of the perceptive mechanism involved in phototropism. Work with algal systems may be of relevance to fungal phototropism. It has, for example, been shown that if part of a Vaucheria filament is illuminated, chloroplasts accumulate at this point. The light inducing this movement is absorbed by the cortical cytoplasm rather than by the chloroplasts themselves (Haupt, 1965). A postulate that a comparable type of system operates in the fungi would explain some of the puzzling features of the light responses which this group show. Various workers have suggested that hyphal growth involves the transport of vesicles containing cell-wall precursors to the apical region of the wall extension (Marchant, Peat \& Banbury, 1967; McClure, Park \& Robinson, 1968; Brenner \& Carroll, 1968; Manocha \& Colvin, 1968). We would postulate that the rate of growth of a hypha is determined by the rate of supply of these vesicles to its apical extension zone. It is possible that under conditions of symmetrical illumination the vesicles are randomly distributed within the conidiophore and are delivered at a more or less equal rate to the whole of the inner surface of the extending hyphal tip. However, when a light gradient is established across a hypha the peripherally located photoreceptor may induce an increase in the rate of supply of vesicles to the wall in the more brightly illuminated half of the cell in a manner similar to chloroplast migration in Vaucheria. Curvatures would thus result from more vesicles being delivered to one part of the extending conidiophore tip than to the other; thus the rate of growth of one side of the conidiophore tip would increase whilst that of the other would decrease by a corresponding amount. This is, of course, exactly what one observes experimentally. We would suppose that the rate of supply of vesicles to the region of the extension zone is governed by factors other than light intensity. This type of model would help to explain how it is possible to have a phototropic response in the absence of any net increase in growth or any demonstrable positive light-growth response. In some respects this hypothesis is similar to that put forward by Castle $(196 \mathrm{I} a, b)$ to explain 'phototropic inversion' and the fact that phototropic bending can be initiated in Phycomyces blakesleeanus without the changes in growth speed that characterize a light-growth response.

It is hoped that future studies using the electron micoscope will help to substantiate this hypothesis.

We are grateful to Dr J. B. Heale for helpful criticism. One of us (A.P.J.T.) wishes to record his thanks to the Central Research Fund of the University of London for a grant towards the cost of the time-lapse photographic equipment. 


\section{REFERENCES}

Banbury, G. H. (1959). Phototropism of lower plants. Ency. Plant. Physiol. 17, 530.

BRenNer, D. M. \& CARroll, G. C. (I968). Fine-structural correlates of growth in hyphae of Ascodesmis sphaerospora. J. Bact. 95, 658.

Carlile, M. J. (1965). The photobiology of fungi. A. Rev. Plant. Physiol. 16, 175.

CASTLE, E.S. (I96I a). Phototropism, adaptation and light-growth responses of Phycomyces. J. gen. Physiol. 45, 39.

Castle, E. S. (1961 b). Phototropic inversion in Phycomyces. Science, N. Y. 133, 1424.

Curry, G. M. \& Gruen, A. E. (1957). Negative phototropism of Phycomyces in the ultraviolet. Nature, Lond. r79, 1028.

DenNison, D. S. (1959). Gallic acid in Phycomyces sporangiophores. Nature, Lond. r84, 2036.

DelBRÜCK, N. \& REICHARDT, W. (1956). I. System analysis for the light growth reactions of Phycomyces. In Cellular Mechanisms in Differentiation and Growth. Ed. by D. Rudnick, pp. 3-44. Princeton University Press.

Gressel, J. B. \& Hartmann, K. M. (1968). Morphogenesis in Trichoderma: Action spectrum of photoinduced sporulation. Planta (Berl.), 79, 27 I.

HAUPT, W. (1965). Perception of environmental stimuli orientating growth and movement in lower plants. A. Rev. Plant Physiol. 16, 267.

LythGoe, J. N. (196I). Effect of light and temperature on growth and development of Thamnidium elegans. Trans. Br. mycol. Soc. 44, 199.

Manocha, M. S. \& Colvin, J. R. (1968). Structure of the cell wall of Pythium debaryanum. J. Bact. 95, 1140.

Marchant, R., Peat, A. \& Banbury, G. H. (1967). The ultrastructural basis of hyphal growth. New Phytol. 66, 623.

McClure, W. K., PARK, D. \& Robinson, P. M. (1968). Apical organization in the somatic hyphae of fungi. J. gen. Microbiol. 50, 177.

MoRoneY, M. J. (1956). Facts from Figures, 3rd edn. London: William Clowes and Sons.

PaGe, R. M. (1956). Studies on the development of asexual reproductive structures in Pilobolus. Mycologia 48, 206.

Shropshire, W. (1963). Photoresponses of the fungus Phycomyces. Physiol. Rev. 43, 38.

Trinci, A. P. J. \& Banbury, G. H. (1967). A study of the growth of tall conidiophores of Aspergillus giganteus. Trans. Br. mycol. Soc. 50, 525.

TrincI, A. P. J. \& BANBury, G. H. (1969). Effect of light on growth and carotenogenesis of the tall conidiophores of Aspergillus giganteus. Trans. Br. mycol. Soc. (in the Press.)

Trinci, A. P. J., Peat, A. \& Banbury, G. H. (1968). Fine structure of phialide and conidiophore development in Aspergillus giganteus Wehmer. Ann. Bot. 32, $24 \mathrm{I}$.

Welty, R. E. \& Christensen, C. M. (1965). Negative phototropic growth of Aspergillus restrictus. Mycologia 57, 311 .

Westaphal, W. H. (1947). Physuik. p. 464. Berlin and Heidelberg: Springer.

\section{EXPLANATION OF PLATE}

Abbreviations used: a, aluminium cap containing water and a cylinder of blotting paper; $c$, culture cell; e, exudation droplet formed under liquid paraffin; o, observation cell; $\mathrm{cn}$, conidiophores.

Fig. I. Culture chamber used to make microscopic observations of conidiophore growth.

Fig. 2. Aspergillus giganteus conidiophores grown at a light intensity of 20 lux.

Fig. 3. A. giganteus conidiophores grown at a light intensity of 200 lux.

Fig. 4. A. giganteus conidiophores unilaterally illuminated with white light whilst submerged in liquid paraffin. The arrow indicates the direction of illumination.

Fig. 5. A. giganteus conidiophores unilaterally illuminated with ultraviolet radiation (280 $\mathrm{m} \mu$ ). The arrow indicates the direction of illumination. 
Journal of General Microbiology, Vol. 54, No. 3

Plate I

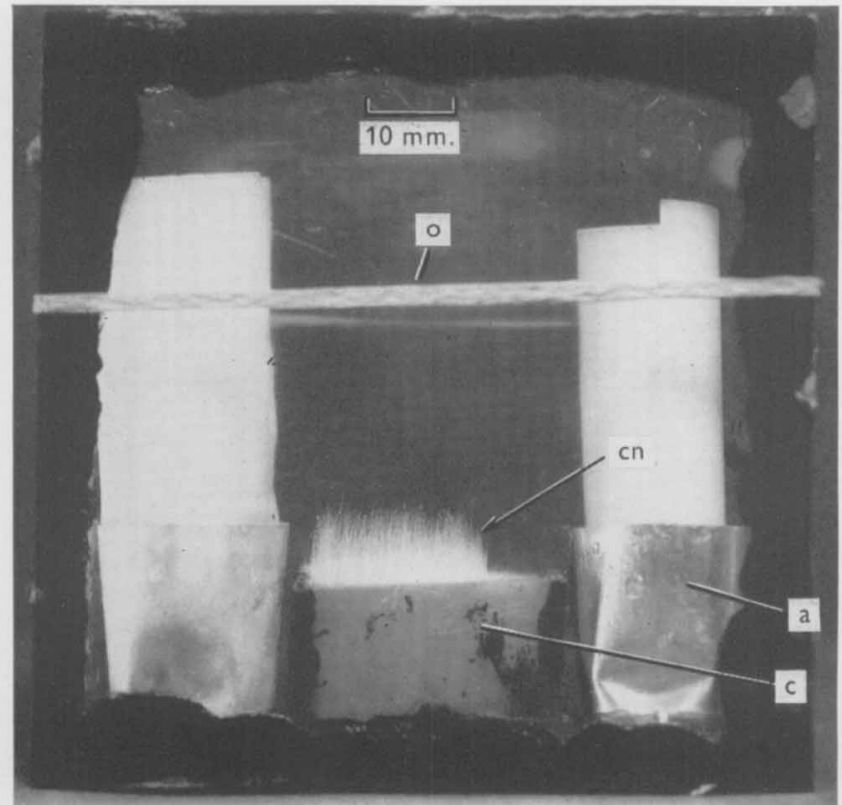

Fig. I

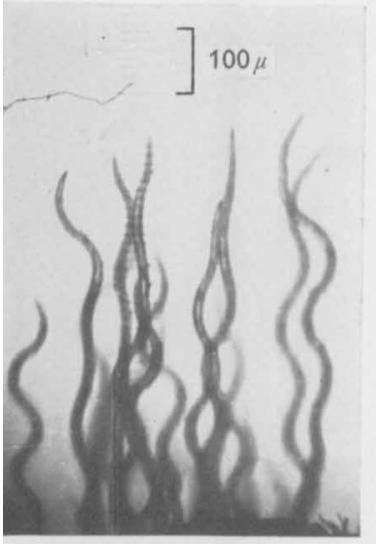

Fig. 2

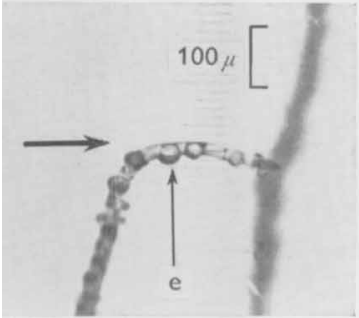

Fig. 4

A. P. J. TRINCI AND G. H. BANBURY

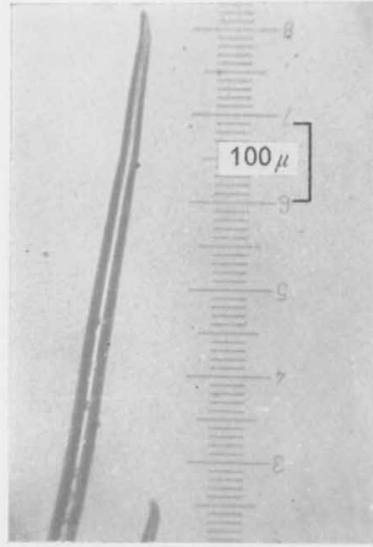

Fig. 3

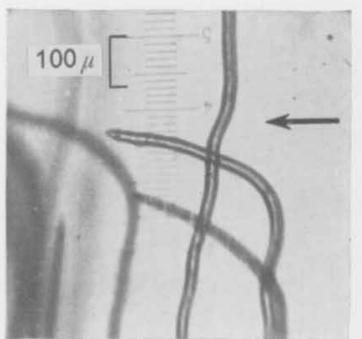

Fig. 5

(Facing p. 438) 